ISSN 0103-5150

Fisioter. Mov., Curitiba, v. 25, n. 3, p. 571-582, jul./set. 2012

Licenciado sob uma Licença Creative Commons

\title{
Prevalência, tipologia e sintomas de gravidade da incontinência urinária em mulheres idosas segundo a prática de atividade física
}

\author{
Prevalence, typology and severity of urinary incontinence \\ symptoms in older women according to physical activity practice
}

\author{
Janeisa Franck Virtuoso ${ }^{[a]}$, Giovana Zarpellon Mazo $^{[\mathrm{b}]}$, Enaiane Cristina Menezes ${ }^{[c]}$ \\ [a] Mestre em Ciências do Movimento Humano pela Universidade do Estado de Santa Catarina (UDESC), Florianópolis, SC - \\ Brasil, e-mail: janeisav@yahoo.com.br \\ [b] Doutora em Ciências do Desporto, professora do Programa de Pós-Graduação em Ciências do Movimento Humano da \\ Universidade do Estado de Santa Catarina (UDESC), Florianópolis, SC - Brasil, e-mail: d2gzm@udesc.br \\ [c] Bolsista de iniciação cientifica da Universidade do Estado de Santa Catarina (UDESC), Florianópolis, SC - Brasil, e-mail: \\ enaianemenezes@gmail.com
}

\section{Resumo}

Introdução: A prática de atividade física (AF) é importante para a população idosa, graças aos beneficios biopsicossociais. A incontinência urinária (IU) também vem sendo analisada, pois o avanço da idade é um fator de risco importante na sua ocorrência. Objetivo: Verificar a prevalência, a tipologia e os sintomas de gravidade da IU entre mulheres idosas segundo a prática de AF regular. Materiais e métodos: Participaram deste estudo 209 idosas, divididas em três grupos, segundo o nível de AF. Foram coletados dados referentes à presença, tipologia, duração e gravidade dos sintomas da IU. Utilizou-se estatística descritiva e inferencial, por meio dos testes Qui-Quadrado, Mann-Whitney e Análise de Variância, conforme os grupos de variáveis. Adotou-se nível de significância de 5\%. Resultados: A presença de IU na amostra total foi de 33,3\%, sendo a menor prevalência entre as idosas mais ativas (28,9\%). Quanto à tipologia, 28,7\% apresentaram IU de esforço (IUE), 14,8\% IU de urgência (IUU) e 10,5\% IU mista (IUM). A presença de IUU $(p=0,05)$ e IUM $(p=0,04)$ associou-se com o grupo de mulheres sedentárias. A prática de ginástica associou-se com a ausência de IU ( $p=0,003)$. Em 43,5\% da amostra, o início dos sintomas de gravidade deu-se após a menopausa. A retenção de urina sem dificuldade associou-se com a prática de AF ( $p=0,029)$. Conclusão: A menor incidência de IU entre as idosas muito ativas pode ser atribuída 
aos benefícios da AF moderada ao mecanismo de continência. Além disso, a prática de exercícios físicos também parece minimizar os sintomas de urgência miccional.

Palavras-chave: Incontinência urinária. Atividade física. Sintomas. Prevalência. Idoso.

\section{Abstract}

Introduction: Physical activity (PA) practice is important for older population considering its biopsychosocial benefits.Urinary Incontinence (UI) has also been investigated since advancing age is an important risk factor to its occurrence. Objective: To investigate prevalence, type and severity of UI symptoms among older women according to regular physical activity practice. Materials and methods: Participants were 209 older women, divided into three groups according to PA level. Data were collected regarding the presence, type, duration and severity of UI symptoms. Descriptive and inferential statistics were used with the Chisquare, Mann-Whitney and ANOVA tests, according to variables' groups. We adopted a significance level of 5\%. Results: The presence of UI in the total sample was 33,3\% and the lowest prevalence was among the more active older women (28.9\%). Regarding the types, 28,7\% had stress UI (SUI), 14,8\% urgency UI (UUI) and $10,5 \%$ mixed UI (MUI). The presence of UUI $(p=0.05)$ and MUI $(p=0.04)$ was associated with the sedentary group. Aerobic gymnastics practice was associated with the absence of UI ( $p=0.003)$. In $43.5 \%$ of the sample the onset of worsening symptoms came after menopause. Retention of urine without difficulties was associated with the practice of PA $(p=0.029)$. Conclusion: The lower incidence of UI among very active older women can be attributed to the benefits of moderate PA in continence mechanism. Moreover, physical exercise also seems to minimize urgency symptoms.

Keywords: Urinary incontinence. Physical activity. Symptoms. Prevalence. Aged.

\section{Introdução}

Segundo a Sociedade Internacional de Continência, incontinência urinária (IU) é definida como a queixa de qualquer perda involuntária de urina. Os tipos mais comuns são: a incontinência urinária de esforço (IUE), que é a perda de urina associada a atividades físicas que aumentam a pressão intra-abdominal; a incontinência urinária de urgência (IUU), perda involuntária de urina associada ao forte desejo de urinar; e a incontinência urinária mista (IUM), em que ambos os tipos anteriores estão presentes (1).

Os índices de prevalência da IU feminina sofrem variação de acordo com a metodologia adotada em cada estudo (2). No entanto, sabe-se que a prevalência de IU costuma ser alta na terceira idade. Estudos internacionais de Mardon et al. (3) e Huang et al. (4) apontam uma prevalência de $44 \%$ e $53 \%$, respectivamente, entre mulheres com 65 anos ou mais.

A presença de IU em mulheres praticantes de exercícios físicos ou esportes tem sido alvo de diversos estudos (5-8). Acredita-se que as atividades de alto impacto podem afetar o mecanismo de continência graças à alteração da quantidade de força transmitida para o assoalho pélvico e pelo aumento excessivo da pressão intra-abdominal (9). Essas alterações comprometem os mecanismos de sustentação, suspensão e contenção do assoalho pélvico, que sofre sobrecarga intensa e repetida, promovendo seu enfraquecimento (5).

Para Nygaard (8), a IUE é o tipo mais frequente entre mulheres fisicamente ativas. Bø e Borgen (10), ao analisar a prevalência de IU entre 660 atletas de elite, observaram que 39\% tinham sintomas de perda urinária ao esforço e apenas 16\%, sintomas de urgência miccional. Nesse sentido, alguns estudos sugerem que a prática de exercícios físicos é um fator de risco importante para o desenvolvimento da IU na mulher $(6,7)$. No entanto, esses estudos são realizados com mulheres jovens e nulíparas, afastando fatores de risco também importantes na gênese da IU, como a idade e a paridade. Poucas pesquisas estudam a população idosa, em que coexistem uma série de outros fatores associados.

Contrapondo-se aos autores mencionados no parágrafo anterior, um estudo realizado por Kikuchi et al. (11), encontrou-se uma prevalência menor de IU entre idosos com alto nível de atividade física 
(16,6\%), quando comparados com idosos com baixo nível $(31,8 \%)$. Assim, embora os resultados deste estudo seccional não possam demonstrar a relação temporal entre a prática de atividade física regular e a presença de IU, os achados indicaram que o exercício físico pode ter um potencial efeito benéfico na prevenção da IU. Bø (12) relata essa hipótese ao sugerir que, simultaneamente ao aumento da pressão abdominal durante o exercício físico, ocorre uma contração reflexa dos músculos do assoalho pélvico. No entanto, sabe-se que mulheres perdem urina durante a atividade física e relatam piora da perda durante a prática, principalmente em atividades de alto impacto.

Soma-se ainda a esse cenário o fenômeno de transição demográfica denominado feminização da velhice, em que as mulheres têm atingido maior longevidade em comparação aos homens, bem como maior presença relativa na população idosa, principalmente nos estratos etários mais velhos (13). Graças a esse fenômeno, nota-se que a presença desse público vem tornando-se maior nos consultórios e clínicas de fisioterapia voltados à saúde da mulher. Além disso, a maior proporção de mulheres idosas em programas de exercícios físicos (14) torna o nível de atividade física uma variável importante na anamnese do fisioterapeuta e demais profissionais da saúde que lidam com esse público.

Considerando-se a problemática supracitada, que expõe a predisposição de mulheres idosas a perdas urinárias, a prática de atividade física que pode ser considerada fator de risco ou de proteção para a ocorrência dessas perdas, bem como a falta de estudos na população idosa, o objetivo deste estudo foi verificar a prevalência, a tipologia e os sintomas de gravidade da incontinência urinária entre mulheres idosas segundo a prática de atividade física regular.

\section{Método}

Tipo de estudo e casuística

Este estudo transversal e descritivo (15) foi realizado com mulheres idosas (60 anos ou mais), que frequentam grupos formais de programas de exercícios físicos para idosos ou que participam de grupos de convivência para idosos, em Florianópolis, SC. 0 processo de seleção da amostra foi realizado de forma intencional, totalizando 209 idosas com idade média de 69,1 $\pm 6,3$ anos.

Para o delineamento amostral, utilizou-se o Domínio 4 do Questionário Internacional de Atividade Física (IPAQ), adaptado para idosos a partir dos estudos de Mazo (16) e Benedetti et al. (17). Esse domínio é composto de três questões e refere-se às atividades físicas de recreação, esporte, exercícios físicos e lazer que o idoso faz em uma semana normal/habitual. São consideradas as atividades físicas com duração de pelo menos dez minutos contínuos e sua intensidade moderada ou vigorosa.

A partir da aplicação desse instrumento, as idosas praticantes foram divididas em Grupo Muito Ativo (GMA $=600$ e $1.500 \mathrm{METs}^{1} /$ minutos por semana) e Grupo Pouco Ativo (GPA = até $600 \mathrm{METs} /$ minutos por semana). As idosas não praticantes foram classificadas como Grupo Sedentário (GSE = zero METs / minutos por semana).

Adotou-se como critério de inclusão no GMA e GPA idosas que praticam exercícios físicos regularmente há pelo menos seis meses em grupos formais de atividades físicas. Já no GSE, selecionaram-se idosas que não praticavam qualquer modalidade de exercício físico por igual período. Foram excluídas de ambos os grupos idosas dependentes para qualquer função, que relataram alguma lesão do trato urinário inferior, presença de dor ao urinar ou qualquer outro indicativo de infecção urinária.

Acrescenta-se, ainda, que, em virtude do número reduzido de participantes na análise dos sintomas de gravidade das mulheres idosas com incontinência urinária ( $\mathrm{n}=69$ ), optou-se por dividir a amostra em dois grupos, segundo a prática regular de atividade física: um grupo composto por mulheres que praticam exercícios físicos regularmente (GMA e GPA) e outro de mulheres que não praticam (GSE). Esses grupos foram chamados de GP (Grupo Praticante) e GNP (Grupo Não Praticante), respectivamente.

Instrumentos e coleta de dados

Para identificar a presença de incontinência urinária na amostra, foi utilizado o relato dos

\footnotetext{
${ }^{1}$ Equivalente metabólico utilizado para quantificar a intensidade da atividade física realizada.
} 
sintomas de perda urinária por meio da questão: Durante o último ano, você perdeu urina (sem querer, na calcinha) pelo menos uma vez no mês? (4). Quando a resposta foi positiva, caracterizou-se como presença de sintomas de incontinência urinária. Se negativo, caracterizou-se como ausência de sintomatologia.

Na presença de sintomas, a tipologia da IU foi identificada por meio das questões:

- Você perde urina quando tosse, espirra, faz força ou carrega peso? Se afirmativo, considerou-se IUE.

- Você perde urina antes de chegar ao banheiro depois de sentir forte vontade de urinar ou sem perceber? Se afirmativo, considerou-se IUU. Quando a idosa respondeu afirmativamente às questões anteriores, considerou-se IUM.

Também foram identificadas a duração e a gravidade das perdas urinárias entre as idosas com sintomatologia positiva, independente da tipologia. As questões foram: tempo de incontinência urinária e período de surgimento; realização de tratamento prévio; dificuldade de chegar ao banheiro no primeiro desejo forte de urinar; quantas vezes vai ao banheiro urinar durante o dia e durante a noite; volume miccional; perda de urina durante o sono; incontinência fecal; situações em que ocorrem as perdas urinárias; quantidade de urina perdida por episódio e o uso de proteção diária (forrinho ou absorvente). Também foi identificada a presença de dor ao urinar, a fim de excluir aquelas idosas com suspeita de infecção urinária. Para as idosas ativas, questionou-se ainda o tempo de prática e a modalidade de exercício.

Para a coleta de dados, fez-se um contato inicial com as idosas para apresentação dos objetivos da pesquisa, a importância da participação, os instrumentos utilizados, o sigilo das informações e o convite para participar. Aquelas que aceitaram foram entrevistadas, de forma individual, antes das aulas do programa de exercício físico ou das atividades propostas pelos grupos de Convivência, no próprio local onde acontecem suas atividades.

\section{Tratamento dos dados}

Os dados coletados foram armazenados em um banco de dados no programa Microsoft ${ }^{\circledR}$ Excel e cada participante foi cadastrada segundo um número codificador. A análise estatística foi realizada no pacote estatístico SPSS - Statistical Package for Social Sciences (versão 17.0).

Inicialmente, todas as variáveis foram analisadas descritivamente a partir de frequência simples e porcentagens (variáveis categóricas) e medidas de posição e dispersão (variáveis numéricas).

Para associação entre variáveis categóricas, utilizou-se o teste de Qui-Quadrado $\left(\chi^{2}\right)$ ou Exato de Fisher, quando necessário. A comparação entre dois grupos, com variáveis numéricas, foi realizada através do teste de Mann-Whitney e entre três grupos com o teste de Análise de Variância (ANOVA - teste post hoc de Bonferoni). Adotou-se um nível de significância de $5 \%$.

\section{Aspectos éticos}

Para realização dessa pesquisa, foram cumpridos os princípios éticos, de acordo com a Resolução n. 196 do Conselho Nacional de Saúde. 0 estudo foi enviado ao Comitê de Ética em Pesquisa da Universidade do Estado de Santa Catarina (UDESC) e aprovado sob protocolo n. 03/2010.

Após concordarem em participar da pesquisa, as idosas assinaram o termo de consentimento em duas vias, ficando uma via de posse da idosa e a outra da pesquisadora responsável.

\section{Resultados}

Com relação aos dados sociodemográficos, as idosas da amostra eram, em sua maioria, casadas $(49,3 \%)$ ou viúvas $(34,9 \%)$, aposentadas $(53,1 \%)$ e com ensino fundamental completo $(30,6 \%)$ ou ensino médio completo $(28,2 \%)$. Conforme as categorias referentes aos níveis de atividade física propostas nesse estudo, 39,7\% foram classificadas como muito ativas (GMA), 33\% como pouco ativas (GPA) e 27,3\% como sedentárias (GSE). Acrescenta-se que a idade não foi uma variável homogênea entre os grupos ( $p=0,003)$, sendo as idosas do GMA mais jovens $(67,3$ $\pm 5,1$ anos) do que aquelas do GPA (70,2 $\pm 6,1$ anos $)$ e GSE $(70,6 \pm 7,4$ anos).

A ocorrência de sintomas de IU, segundo os grupos definidos a partir do nível de atividade física, pode ser observada na Tabela 1 . 
Tabela 1 - Associação entre a sintomatologia da incontinência urinária e os grupos, segundo o nível de atividade física muito ativa, pouco ativa e sedentária $(n=209)$

\begin{tabular}{|c|c|c|c|c|c|c|}
\hline \multirow[t]{2}{*}{ Variáveis } & $\begin{array}{c}\text { GMA } \\
(n=83)\end{array}$ & $\begin{array}{c}\text { GPA } \\
(n=69)\end{array}$ & $\begin{array}{c}\text { GSE } \\
(n=57)\end{array}$ & $\begin{array}{c}\text { Total } \\
(\mathrm{n}=209)\end{array}$ & \multirow[t]{2}{*}{$x^{2}$} & \multirow[t]{2}{*}{$\mathrm{p}$} \\
\hline & f (\%) & $f(\%)$ & $f(\%)$ & f (\%) & & \\
\hline Presença de IU & $24(28,9)$ & $25(36,2)$ & $20(35,1)$ & $69(33,3)$ & 1,06 & 0,590 \\
\hline Presença de IUE & $21(25,3)$ & $22(31,9)$ & $17(29,8)$ & $60(28,7)$ & 0,84 & 0,650 \\
\hline Presença de IUU & $9(11,0)$ & $8(11,6)$ & $14(24,6)^{*}$ & $31(14,9)$ & 5,78 & $0,050^{*}$ \\
\hline Presença de IUM & $6(7,2)$ & $5(7,2)$ & $11(19,3)^{*}$ & $22(10,5)$ & 6,40 & $0,040^{*}$ \\
\hline
\end{tabular}

Fonte: Dados da pesquisa.

Legenda: IU = incontinência urinária; IUE = incontinência urinária de esforço; IUU = incontinência urinária de urgência; IUM = incontinência urinária mista; GMA = grupo muito ativa; GPA = grupo pouco ativa; GSE = grupo sedentária; $x^{2}=$ teste Qui-Quadrado; $p=$ nível de significância; $¥=$ ajuste residual $\geq 2,0 ;{ }^{*} p \leq 0,05$.

A presença de incontinência urinária na amostra foi de $33,3 \%$, sendo $28,7 \%$ com sintomas de incontinência urinária de esforço (IUE), 14,8\% incontinência urinária de urgência (IUU) e 10,5\% incontinência de sintomas mistos (IUM). A associação entre a presença de IUU e IUM e os grupos segundo o nível de atividade física foi significativa ( $p=0,05$ e $p=0,04$, respectivamente). Conforme o ajuste residual $\left({ }^{\ddagger}\right)$, há uma tendência de as mulheres idosas sedentárias (GSE) apresentarem IUU e IUM.

0 tempo de prática de atividade física no GMA e GPA físicas foi de 3,99 $\pm 6,85$ anos e de 2,04 $\pm 2,50$ anos, respectivamente, havendo diferença significativa entre os grupos $(p=0,028)$. No entanto, não houve diferença entre idosas continentes e incontinentes ( GMA $=0,505$ e GPA $=0,158$ anos), sugerindo que o tempo de prática de atividade física não influenciou a gênese da IU nos grupos praticantes.

Com relação às modalidades de exercício físico, a maioria das idosas ativas (GMA e GPA) pratica ginástica (82,9\%), seguida da caminhada $(38,8 \%)$, hidroginástica $(14,5 \%)$ e outras, com menor percentagem (Figura 1).

A ginástica foi a única modalidade que se associou com a incontinência urinária $\left(\chi^{2}=4,530 ; p=\right.$ $0,003)$. Conforme o ajuste residual $(2,1)$, a prática de ginástica associou-se com a ausência de incontinência urinária, revelando que $71,4 \%$ das idosas que praticam ginástica não sofrem esse sintoma.

0 número de modalidades praticadas pelas idosas ativas (GMA e GPA) pode ser observado na Figura 2. A maioria pratica apenas uma modalidade $(52,0 \%)$, enquanto $39 \%$ praticam duas modalidades e $9 \%$ praticam três. Não houve associação entre o número de modalidades a ocorrência de IU.

Com relação aos sintomas de gravidade entre as idosas incontinentes, foi possível observar que, para a maioria (43,5\%), o início dos sintomas deu-se após a menopausa; $29 \%$ não se recordam de qualquer fato gerador e apenas 8,7\% relataram o início dos sintomas após o parto. Aproximadamente, $25 \%$ da amostra já procuraram tratamento clínico para solucionar o quadro de perdas urinárias, sendo que nove (13\%) realizaram procedimento cirúrgico e cinco, (7,2\%) farmacológico.

Quanto ao ato miccional, três $(4,3 \%)$ relataram dificuldade de iniciá-lo e mais da metade $(52,2 \%)$ considerava seu volume miccional médio. A perda de urina durante o sono $(11,6 \%)$ e a incontinência fecal $(5,8 \%)$ foram mencionadas por uma minoria da amostra.

Conforme proposto na metodologia, em função do número reduzido de mulheres com incontinência urinária $(n=69)$, a amostra foi dividida em dois grupos: um composto por mulheres que praticam exercícios físicos regularmente (GMA e GPA), chamado de Grupo Praticante (GP), e outro, de mulheres que não praticam (GSE), intitulado como Grupo Não Praticante (GNP).

Na Tabela 2, apresentam-se algumas medidas de gravidade, segundo os grupos praticantes e não praticantes.

As medidas de gravidade, observadas na Tabela 2, são semelhantes entre as idosas incontinentes do GP 


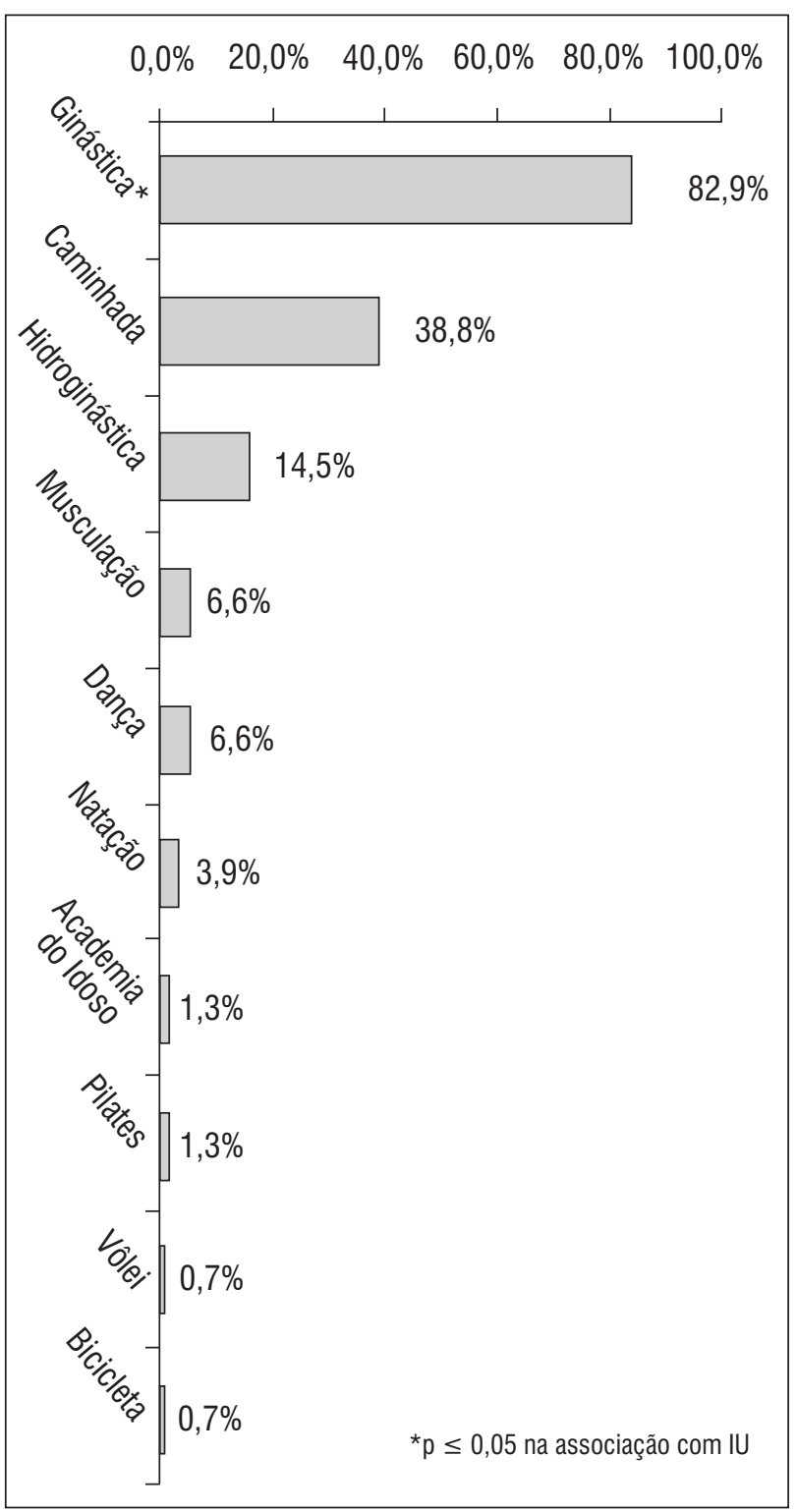

Figura 1 - Modalidades de exercício físico praticadas pelas idosas da amostra $(n=152)$

Fonte: Dados da pesquisa.

e GNP, com exceção da retenção urinária ao desejo forte de urinar $(p=0,029)$, havendo uma tendência do grupo praticante de exercícios físicos em reter a urina sem dificuldade $(55,1 \%)$. Também houve uma tendência desse grupo em não utilizar absorventes, porém, sem associação significativa ( $p=0,073$ ).

Entre as idosas praticantes, apenas sete $(14,3 \%)$ declararam perda urinária durante o exercício físico, sendo que quatro mencionaram perda em gotas e três em forma de jato. Para manter-se seca e por precaução, o dobro de mulheres (14) utilizava absorvente durante o exercício físico.

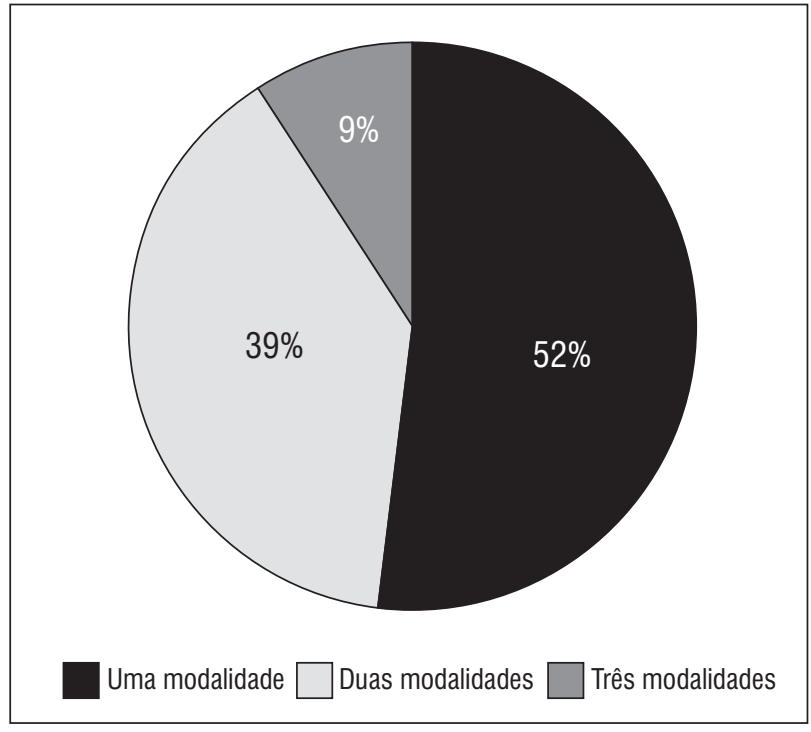

Figura 2 - Número de modalidades de exercício físico praticadas pelas idosas da amostra $(n=152)$

Fonte: Dados da pesquisa.

As atividades de perda urinária citadas pelas idosas da amostra (GP e GNP) estão expostas na Figura 3.

Observa-se que tossir e espirrar foram as atividades motivadoras de maior incidência de perda urinária na amostra. As atividades menos mencionadas foram: levantar da posição deitada (8,7\%), situações de frio $(8,7 \%)$, situações de estresse $(8,7 \%)$, orgasmo $(4,3 \%)$, levantar da posição sentada $(4,3 \%)$ e barulho da torneira $(4,3 \%)$. Não houve diferença significativa entre a freqüência dessas atividades de perda urinária e os grupos praticantes e não praticantes de exercício físico.

\section{Discussão}

Quanto à tipologia, a maioria das idosas relatou perda urinária aos esforços (28,7\%), enquanto 14,9\% apresentaram incontinência urinária de urgência e apenas $10,5 \%$, incontinência de sintomas mistos. Tannenbaum, Corcos e Assalian (18) encontraram incidência similar de IUE entre mulheres com 55 anos ou mais (32\%), no entanto, os valores de IUU (22\%) e IUM (32\%) foram superiores.

Barros, Lucena e Anselmo (19) e Mourão et al. (20) explicam que, em virtude da deficiência de estrógenos, o trato urinário inferior feminino apresenta algumas alterações, tal como a diminuição da força de contração dos músculos do assoalho pélvico, 
Tabela 2 - Comparação/associação entre as medidas de gravidade da incontinência urinária e os grupos praticantes e não praticantes de exercícios físicos $(n=69)$

\begin{tabular}{|c|c|c|c|c|c|}
\hline & $\begin{array}{c}\text { GP } \\
(n=49)\end{array}$ & $\begin{array}{c}\text { GNP } \\
(n=20)\end{array}$ & $\begin{array}{c}\text { Total } \\
(n=69)\end{array}$ & & \\
\hline Variáveis Numéricas & & & & $\mathbf{U}$ & $\mathrm{p}$ \\
\hline 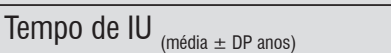 & $8,06 \pm 10,10$ & $7,85 \pm 11,97$ & $8,00 \pm 10,61$ & 470,0 & 0,790 \\
\hline Idas ao banheiro/dia (mediana (I0)) & $5(3)$ & $4,5(2)$ & $5(2)$ & 484,0 & 0,935 \\
\hline Idas ao banheiro/noite (mediana (l0)) & 2 (2) & 1 (2) & 2 (2) & 389,0 & 0,167 \\
\hline Variáveis Categóricas & $f(\%)$ & $f(\%)$ & $f(\%)$ & $\mathrm{X}^{2}$ & $\mathrm{p}$ \\
\hline \multicolumn{6}{|l|}{ Desejo forte de urinar } \\
\hline Retém com dificuldade & $17(34,7)$ & $12(60,0)$ & $29(42,0)$ & \multirow{3}{*}{7,104} & \multirow{3}{*}{$0,029 *$} \\
\hline Retém sem dificuldade & $27(55,1)^{*}$ & $4(20,0)$ & $31(44,9)$ & & \\
\hline Não consegue reter & $5(10,2)$ & $4(20,0)$ & $9(13,0)$ & & \\
\hline \multicolumn{6}{|l|}{ Uso de absorvente } \\
\hline Sempre & $14(28,6)$ & $7(35,0)$ & $21(30,4)$ & \multirow{3}{*}{5,237} & \multirow{3}{*}{0,073} \\
\hline Ocasionalmente & $14(28,6)$ & $10(50,0)$ & $24(34,8)$ & & \\
\hline Nunca & $21(42,9)^{*}$ & $3(15,0)$ & $24(34,8)$ & & \\
\hline \multicolumn{6}{|l|}{ Quantidade de perda urinária } \\
\hline Algumas gotas & $29(59,2)$ & $12(60,0)$ & $41(59,4)$ & 0,004 & 0,950 \\
\hline Jato & $20(40,8)$ & $8(40,0)$ & $28(40,6)$ & & \\
\hline
\end{tabular}

Fonte: Dados da pesquisa.

Legenda: IU = Incontinência Urinária; GP = Grupo Praticante; GNP = Grupo Não Praticante; $I Q$ = Distância Interquartilica; $f$ = frequência; $U$ = teste $U$ de Mann-Whitney; $x^{2}=$ teste Qui-Quadrado; $p=$ nível de significância; $¥=$ ajuste residual $\geq 2,0$; ${ }^{*} \leq \leq 0,05$.

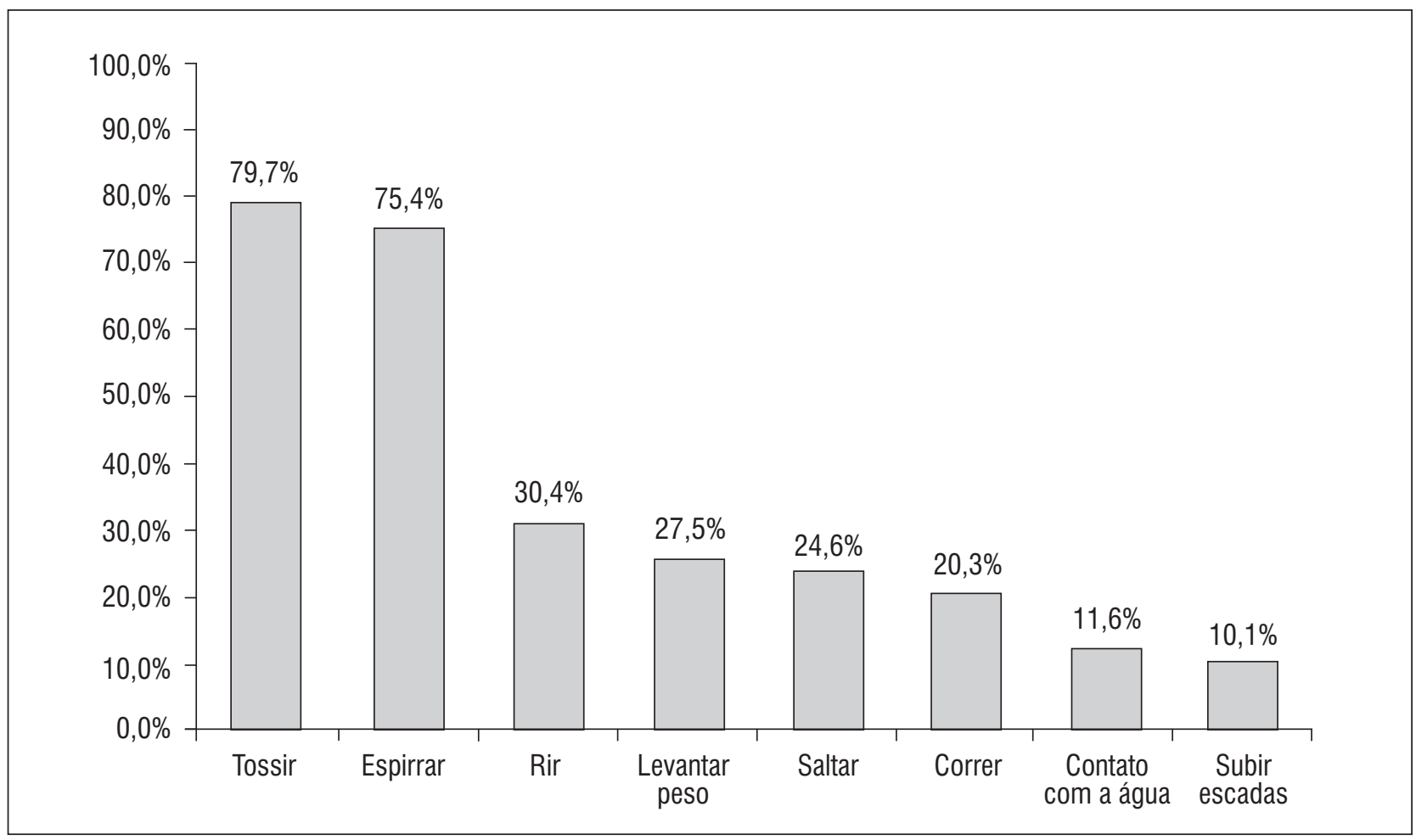

Figura 3 - Atividades de perda urinária relatada pelas idosas incontinentes da amostra $(n=69)$ Fonte: Dados da pesquisa. 
que podem provocar a perda involuntária de urina mediante esforços. Além disso, com o processo de envelhecimento, alterações estruturais do músculo detrusor, como desenvolvimento de fibroses e hipersensibilidade à noradrenalina, resultam em redução da capacidade vesical e desenvolvimento de contrações involuntárias (21), provocando sintomas de urgência miccional e/ou IUU.

Com relação ao nível de atividade física, nota-se que as idosas pouco ativas (GPA) apresentaram maior incidência de perdas urinárias $(36,2 \%)$, seguido do grupo de sedentárias (GSE, 35,1\%) e o grupo de mulheres muito ativas (GMA, 28,7\%). Smith et al. (22) encontraram associação entre a prática de exercícios físicos e menores taxas de IU. Conforme os autores, exercícios de intensidade moderada devem ser incentivados, a fim de promover a continência urinária. Ree, Nygaard e Bø (23) justificam esses achados quando afirmam que o exercício físico pode aumentar o volume dos músculos do assoalho pélvico, tornando-os capazes de contrair-se durante o aumento da pressão intra-abdominal, diminuindo os episódios de IU.

Nesse contexto, observa-se que o grupo de mulheres que praticava ginástica, atividade considerada de intensidade moderada, apresentou menores taxas de IU ( $\mathrm{p}<0,05)$. Segundo Danforth et al. (24), a atividade física está associada com uma significante redução da IU entre mulheres idosas, sendo que os resultados estão mais relacionados com a IUE do que com a IUU. Hay-Smith et al. (25) revela, na última publicação da ICI (International Consultation Incontinence), que o exercício físico de baixa e moderada intensidade diminui a incidência de IU em mulheres de meia idade e idosas, sendo classificado como nível de evidência 2 , ou seja, existem bons estudos de coorte referentes a essa informação.

No entanto, a prática de exercícios físicos extenuantes tem sido indicada como um fator de promoção de disfunções do assoalho pélvico $(12,26)$. Para Kikuchi et al. (11), atividades físicas vigorosas podem levar ao aumento da pressão intra-abdominal, tornando-se um importante fator associado com a ocorrência de IU. Nygaard et al. (8) e Bø (12) ainda afirmam que a prática esportiva profissional constitui um risco adicional ao desenvolvimento da IUE, principalmente quando relacionada à atividades de alto impacto. Entende-se como atividade de alto impacto aquela que ocasiona aumento excessivo da pressão intra-abdominal, sobrecarregando os órgãos pélvicos e empurrando-os para baixo, como, por exemplo, atividades com saltos e aterrissagens e, também, corridas (9).

A constituição dos grupos, segundo o nível de atividade física, pode explicar parcialmente a maior incidência de IU entre as mulheres do GPA. Conforme consta na metodologia, o GMA foi composto por idosas que se enquadraram como Alto Nível e Nível Moderado, já que apenas quatro idosas foram classificadas como Alto Nível, enquanto o GPA foi constituído por mulheres categorizadas como Baixo Nível. Nota-se, portanto, a ocorrência de menores taxas de IU entre idosas com maior nível de atividade física, reiterando os autores mencionados no parágrafo anterior.

Com relação à associação entre o tipo de IU e os grupos, nota-se a tendência de as idosas sedentárias apresentarem sintomas de urgência $(\mathrm{p}=$ 0,050 ). A média de idade superior nesse grupo pode justificar esse quadro. Além disso, em estudo transversal, Song et al. (27) observaram que as mulheres que se exercitavam pelo menos uma vez por semana eram menos propensas a apresentar incontinência urinária por urgência. Townsend et al. (28) também encontrou menores taxas de IUU em mulheres com maior nível de atividade física (OR=0,53; IC 95\% = 0,31 a 0,90 ). Esses resultados demonstram que os sintomas de urgência miccional também podem ser amenizados com a prática regular de exercícios físicos. Complementa-se ainda, nesse contexto, que há associação significativa entre as idosas incontinentes do GP e a retenção urinária sem dificuldade ao forte desejo de urinar (Tabela 2).

No que se refere aos sintomas de gravidade, nota-se que a maioria das idosas incontinentes percebeu o início dos sintomas com a chegada da menopausa. Sánchez e Cevallos (29) afirmam que, para manter a continência urinária, a pressão uretral deve permanecer estável, a partir da ação do esfíncter uretral interno e externo, da união uretrovesical e das vias neurológicas intactas. Todas essas estruturas apresentam uma expressiva densidade de receptores de estrogênios (30), portanto, a redução desse hormônio durante a menopausa pode levar ao quadro de perdas urinárias, conforme demonstrado na literatura referente ao tema $(31,32)$.

A incontinência urinária é erroneamente interpretada como parte natural do envelhecimento (31). Algumas mulheres desvalorizam o sintoma, quando o incômodo causado pela incontinência é pequeno, 
pois acham que isso não justifica consulta ao médico (33). Em um inquérito domiciliar, realizado em mulheres incontinentes com idade variando de 45 a 60 anos, observou-se que $54 \%$ não consultaram um médico, pois acreditavam que os sintomas não mereciam atenção, $14 \%$ afirmaram que os sintomas não eram tão fortes ou não incomodavam e $9 \%$ não sentiam liberdade de falar desses sintomas com o médico (34). Em outro estudo realizado com mulheres profissionais de enfermagem, $79 \%$ das incontinentes não procuraram tratamento médico, sendo os principais motivos a pequena quantidade de urina perdida $(28,8 \%)$ e a crença de que a IU é um problema comum entre as mulheres (22\%) (33). Semelhante ao encontrado nesse último estudo, 75\% das idosas entrevistadas na presente pesquisa não haviam procurado tratamento para o seu quadro de perdas urinárias. 0 tempo médio, em anos, em que essas mulheres apresentam esses sintomas $(8,00 \pm$ $10,61)$ reitera a desvalorização do sintoma entre as participantes da amostra.

Quanto à perda involuntária de urina durante o sono, denominada enurese noturna (35), nota-se que $11,6 \%$ da amostra relatou essa queixa. A noctúria, ato de acordar para urinar uma ou mais vezes durante a noite (36), costuma ser o tipo mais analisado entre os sintomas miccionais noturnos na população idosa. Um estudo realizado por Mourão et al. (20) com mulheres a partir de 40 anos, participantes de grupos de atividades físicas, encontraram uma prevalência de enurese noturna de $52,38 \%$. No entanto, a casuística do estudo foi muito pequena (50 mulheres, sendo que $42 \%$ tinham sintomas de incontinência urinária), dificultando a comparação com esse presente estudo.

Já com relação à noctúria, observa-se, na Tabela 2 , que a mediana de idas ao banheiro à noite da amostra total de mulheres incontinentes foi 2 , sugerindo que, além dos sintomas de incontinência urinária, essas mulheres acordam durante a noite para urinar, prejudicando a qualidade do sono (37). Conforme Reis et al. (31), essa é uma das alterações mais frequentes do hábito urinário no idoso, pois alterações hormonais decorrentes do envelhecimento, como o aumento na secreção de vasopressina e do hormônio natriurético, podem resultar na eliminação preponderante dos líquidos ingeridos (durante o dia) no período noturno.

Quanto à presença de incontinência fecal (IF), apenas 5,8\% das mulheres incontinentes mencionaram tal queixa. Segundo Leung e Rao (38), aproximadamente $20 \%$ da comunidade idosa apresenta esses sintomas. A prevalência aumenta entre idosos residentes em instituições de longa permanência (50\%). A IF é caracterizada pela fraqueza do assoalho pélvico e da musculatura esfincteriana do canal anal. Assim como os demais músculos do corpo, essas estruturas estão sujeitas à interferência de processos degenerativos. Dessa forma, aponta-se, além do número elevado de partos vaginais, o processo de envelhecimento como principal causa dessa deficiência esfincteriana (39).

Com relação à perda de urina durante o exercício físico, apenas sete $(14,3 \%)$ relataram essa queixa. Nygaard et al. (40) também encontraram valores semelhantes, ao verificarem a prevalência de perda de urina entre mulheres de meia idade. Os autores constataram que $11,8 \%$ das mulheres tinham perdas enquanto caminhavam, 15,1\% em atividades físicas moderadas e $15,9 \%$, em atividades vigorosas. Nota-se que as idosas, com queixa de IU durante o exercício físico, praticavam ginástica. A baixa incidência de perdas urinárias durante o exercício pode ser justificada por tratar-se de uma modalidade de baixa a moderada intensidade.

A IU afeta diretamente a qualidade de vidas das mulheres, exercendo múltiplos efeitos sobre as atividades diárias, interações sociais e percepção de saúde (41). Em uma pesquisa realizada por Lopes e Higa (42) com 164 mulheres incontinentes, observou-se importante restrição das atividades sociais $(33,5 \%)$, sendo que a IU impedia a mulher de sair de casa $(27,4 \%)$, ir a festas e ao clube $(6,7 \%)$, fazer viagens longas $(5,5 \%)$, frequentar a igreja $(4,3 \%) \mathrm{e}$ participar atividades físicas $(3,7 \%)$. 0 estudo ainda revela que as mulheres que deixavam de sair de casa admitiam medo ou vergonha de perder urina em público e de ficar molhadas, de exalar odor de urina, e de não encontrarem banheiro em caso de emergência. Diante dessas exposições, sugere-se que o número reduzido de idosas com perdas urinárias durante o exercício físico também pode refletir o abandono de atividades físicas e sociais em virtude dessas restrições causadas pela IU.

\section{Considerações finais}

Sendo a idade e o sexo feminino fatores de risco importantes no desenvolvimento da incontinência urinária (IU), a presença desse sintoma na amostra 
total, composta por mulheres idosas, foi semelhante à da literatura específica. A menor incidência entre as idosas muito ativas (GMA) pode ser atribuída aos benefícios da atividade física moderada no mecanismo de continência urinária. Além disso, a ginástica associou-se significantemente com a ausência de IU. A incontinência urinária de esforço (IUE) foi o tipo mais comum, seguido da incontinência urinária de urgência (IUU) e incontinência urinária mista (IUM). Apenas os dois últimos tipos associaram-se com os grupos segundo o nível de atividade física.

Quanto aos sintomas de gravidade, nota-se que o advento da menopausa é considerado um fato propiciador da IU para a maioria das idosas. Os sintomas de gravidade foram semelhantes entre idosas praticantes (GP) e não praticantes de exercícios físicos (GNP), havendo associação somente entre o GP e a retenção de urina sem dificuldade. Diante desses resultados e da associação significativa entre a IUU e o GSE, sugere-se que a prática de exercícios físicos pode minimizar, também, os sintomas de urgência miccional.

A partir dos resultados encontrados no presente estudo, fisioterapeutas e demais profissionais da saúde podem recomendar a prática de exercícios físicos moderados para suas pacientes/clientes idosas, a fim de prevenir e minimizar a queixa de perdas urinárias.

Sugere-se que estudos futuros avancem na composição de grupos mais homogêneos com relação à idade, uma vez que se trata de uma variável importante na gênese da IU. Além disso, recomenda-se a utilização de todos os domínios do Questionário Internacional de Atividade Física (IPAQ), com vistas a identificar o nível de atividade física em outras situações, como atividades domésticas e de deslocamento.

\section{Referências}

1. Abrams P, Cardozo L, Fall M, Griffiths D, Rosier P, Ulmsten $\mathrm{U}$, et al. The standardization of terminology of lower urinary tract function: report from the standardization sub-committee of the international continence society. Urology. 2003;61(1):37-49. doi:10.1016/ S0090-4295(02)02243-4.

2. Higa R, Lopes MHBM, Reis MJ. Fatores de risco para incontinência urinária na mulher. Rev Esc Enferm USP. 2008;42(1):187-92. doi:10.1590/ S0080-62342008000100025.
3. Mardon RE, Halim S, Pawlson LG, Haffer SC. Management of urinary incontinence in Medicare managed care beneficiaries: results from the 2004 Medicare Health Outcomes Survey. Arch Intern Med. 2006;166(10):112833. doi:10.1001/archinte.166.10.1128.

4. Huang AJ, Brown JS, Thom DH, Fink HA, Yaffe K. Urinary incontinence in older community-dwelling women: the role of cognitive and physical function decline. Obstet Gynecol. 2007;109(4):909-16. doi:10.1097/01. AOG.0000258277.01497.4b.

5. Chiarapa TR, Cacho DP, Alves AFD. Incontinência urinária feminina: assistência fisioterapêutica e multidisciplinar. São Paulo: Livraria Médica Paulista; 2007. p. 71-122.

6. Nygaard I, Thompson FL, Svengalis SL, Albright JP. Urinary incontinence in elite nulliparous athletes. Obstet Gynecol. 1994;84(2):183-7. PMid:8041527.

7. Thyssen HH, Clevin L, Olesen S, Lose G. Urinary incontinence in elite female athletes and dancers. Int Urogynecol J Pelvic Floor Dysfunct. 2002;13(1):15-7. doi:10.1007/ s001920200003.

8. Nygaard I. Does prolonged high-impact activity contribute to later urinary incontinence? A retrospective cohort study of female Olympians. Obstet Gynecol. 1997;90(5):71822. doi:10.1016/S0029-7844(97)00436-5.

9. Caetano AS, Tavares MCGCF, Lopes MHBM. Urinary incontinence and physical activity practice. Rev Bras Med Esporte. 2007;13(4):270-4. doi:10.1590/ S1517-86922007000400012.

10. Bø K, Borgen JS. Prevalence of stress and urge urinary incontinence in elite athletes and controls. Med Sci Sports Exerc. 2001;33(11):1797-802. doi:10.1097/00005768-200111000-00001.

11. Kikuchi A, Niu K, Ikeda Y, Hozawa A, Nakagawa H, Guo H, et al. Association between physical activity and urinary incontinence in a community-based elderly population aged 70 years and over. Eur Urol. 2007;52(3):868-74. doi:10.1016/j.eururo.2007.03.041.

12. Bø K. Urinary incontinence, pelvic floor dysfunction, exercise and sport. Sports Med. 2004;34(7):451-64. doi:10.2165/00007256-200434070-00004.

13. Neri AL. Idosos no Brasil vivências, desafios e expectativas na terceira idade. São Paulo: Fundação Perseu Abramo; 2007.

14. Andreotti MC, Okuma SS. Perfil sócio demográfico e de adesão inicial de idosos ingressantes em um programa de educação física. Rev Paul Educ Fís. 2003;17(2):142-53. 
15. Gil AC. Como elaborar projetos de pesquisa. 4a ed. São Paulo: Atlas; 2009.

16. Mazo GZ. Atividade física e qualidade de vida de mulheres idosas [tese]. Portugal: Universidade do Porto, Faculdade de Ciências do Desporto e de Educação Física; 2003.

17. Benedetti TB, Mazo GZ, Barros MVG. Aplicação do questionário internacional de atividades físicas para avaliação do nível de atividades físicas de mulheres idosas: validade concorrente e reprodutibilidade teste-reteste. Rev Bras Ciênc Mov. 2004;12(1):25-34.

18. Tannenbaum C, Corcos J, Assalian P. The relationship between sexual activity and urinary incontinence in older women. J Am Geriatr Soc. 2006;54(8):1220-4. doi:10.1111/j.1532-5415.2006.00827.x.

19. Barros JD, Lucena ACT, Anselmo CWSF. Incontinência urinária de esforço em atletas do sexo feminino: uma revisão da literatura. An Fac Med Univ Fed Pernamb. 2007;52(2):173-80.

20. Mourão FAG, Lopes LN, Vasconcellos NPC, Almeida MBA. Prevalência de queixas urinárias e o impacto destas na qualidade de vida de mulheres integrantes de grupos de atividade física. Acta Fisiatr. 2008;15(3):170-5.

21. Siroky MB. The aging bladder. Rev Urol. 2004;6 Suppl 1:S3-7.

22. Smith AL,WangPC,AngerJT,MangioneCM,TrejoL,Rodríguez $\mathrm{LV}$, et al. Correlates of urinary incontinence in communitydwelling older latinos. J Am Geriatr Soc. 2010;58(6):11706. doi:10.1111/j.1532-5415.2010.02814.x.

23. Ree ML, Nygaard I, Bø K. Muscular fatigue in the pelvic floor muscles after strenuous physical activity. Acta Obstet Gynecol Scand. 2007;86(7):870-6. doi:10.1080/00016340701417281.

24. Danforth KN, Shah AD, Townsend MK, Lifford KL, Curhan GC, Resnick NM, et al. Physical activity and urinary incontinence among healthy, older women. Obstet Gynecol. 2007;109(3):721-7. doi:10.1097/01. AOG.0000255973.92450.24.

25. Hay Smith J, Nygaard I, Wyman J, Yamanishi T, Berghmans B, Hagen S, et al. Adult conservative management. In: Abrams P, Cardozo L, Khoury S, Wein A. Incontinence 4th International Consultation on Incontinence. Paris: Health Publication Ltd; 2009. p. 1025-120.

26. Wilson PD, Berghmans B, Hagen S, Hay-Smith J, Moore $\mathrm{K}$, Nygaard I, et al. Adult conservative treatment. In: Abrams P, Cardozo L, Khoury S, Wein A. Incontinence. Management. Plymouth: Health Publication Ltd; 2005. p. 855-964.
27. Song YF, Zhang WJ, Song J, Xu B. Prevalence and risk factors of urinary incontinence in Fuzhou Chinese women. Chin Med J (Engl). 2005;118(11):887-92.

28. Townsend MK, Danforth KN, Rosner B, Curhan GC, Resnick NM, Grodstein F. Physical activity and incident urinary incontinence in middle-aged women. J Urol. 2008;179(3):1012-6. doi:10.1016/j.juro.2007.10.058.

29. Sánchez R, Cevallos C. Incontinencia urinaria en el climatério. Rev del Climatério. 2006;9(50):56-61.

30. Aldrighi JM, Aldrighi CMS, Aldrighi APS. Alterações sistêmicas do climatério. Rev Bras Med. 2002;1(1):15 21. doi:10.1590/S0104-42302002000100019.

31. Reis RB, Cologna AJ, Martins ACP, Paschoalin EL, Tucci $\mathrm{S}$ Jr, Suaid HJ. Incontinência urinária no idoso. Acta Cir Bras. 2003;18(Supl 5):47-51.

32. Berlezi EM, Dal Bem A, Antonello C, Leite MT, Bertolo EM. Incontinência urinária em mulheres no período pós-menopausa: um problema de saúde pública. Rev Bras Geriatr Gerontol. 2009;12(2):159-73.

33. Higa R, Lopes MHBM. Porque profissionais de enfermagem com incontinência urinária não buscam tratamento. Rev Bras Enferm. 2007;60(5):503-6. doi:10.1590/ S0034-71672007000500004.

34. Guarisi T, Pinto Neto AM, Osis MJ, Pedro AO, Paiva LHSC, Faúndes A. Procura de serviço médico por mulheres com incontinência urinária. Rev Bras Ginecol Obstet. 2001;23(7):439-43.

35. Alóe F, Biagini JC Jr, Tavares S. Parassonias: diagnóstico e tratamento. Diagn Tratamento. 2002;7(3):22-9.

36. Van Kerrebroeck P, Abrams P, Chaikin D, Donovan J, Fonda D, Jackson S, et al. The standardisation of terminology in nocturia; report from the standardisation sub-committee of the International Continence Society. Neurourol Urodyn. 2002;21(2):179-83. doi:10.1002/nau.10053.

37. Bliwise DL, Foley DJ, Vitiello MV, Ansari FP, AncoliIsrael S, Walsh JK. Nocturia and disturbed sleep in the elderly. Sleep Med. 2009;10(5):540-8. doi:10.1016/j. sleep.2008.04.002.

38. Leung FW, Rao SS. Fecal incontinence in the elderly. Gastroenterol Clin North Am. 2009;38(3):503-11. doi:10.1016/j.gtc.2009.06.007.

39. Lorenzentti F, Dambros M, Correa RS. Fisiopatologia da incontinência fecal. In: Palma P. Urofisioterapia: aplicações clínicas das técnicas fisioterapêuticas nas disfunções miccionais e do assoalho pélvico. Campinas: Personal Link Comunicações; 2009. p. 121-8. 
40. Nygaard I, Girts T, Fultz NH, Kinchen K, Pohl G, Sternfeld B. Is urinary incontinence a barrier to exercise in women? Obstet Gynecol. 2005;106(2):307-14. doi:10.1097/01. AOG.0000168455.39156.0f.

41. Dedicação AC, Haddad M, Saldanha MES, Driusso P. Comparação da qualidade de vida nos diferentes tipos de incontinência urinária feminina. Rev Bras Fisioter. 2008;13(2):116-22.
42. Lopes MHBM, Higa R. Restrições causadas pela incontinência urinária à vida da mulher. Rev Esc Enferm USP. 2006;40(1):34-41. doi:10.1590/S008062342006000100005 .

Recebido: 04/05/2011

Received: 05/04/2011

Aprovado: 04/01/2012

Approved: 01/04/2012 